\title{
Spatial proximity as a determinant of context-specific attentional settings
}

\author{
Nathaniel T. Diede ${ }^{1}$ - Julie M. Bugg ${ }^{1}$
}

Published online: 16 March 2016

(C) The Psychonomic Society, Inc. 2016

\begin{abstract}
People implicitly encode the history of conflict associated with particular contexts and use this information to modulate attention to distractors. This manifests as a reduction in the compatibility effect in mostly incompatible locations compared to mostly compatible locations, a difference termed the context-specific proportion compatibility (CSPC) effect. CSPC effects are explained by an episodic retrieval account positing that abstract attentional settings bind to contextual cues - allowing rapid, context-driven modulation of attention. The current study interrogated this binding process by testing the influence of relative spatial proximity on the association of attentional settings with particular locations. In Experiment 1, like typical CSPC paradigms, biased locations appeared near top (e.g., mostly compatible) and bottom (e.g., mostly incompatible) edges of the screen. A novel feature was the addition of two mostly compatible (above fixation) and two mostly incompatible (below fixation) locations placed within close proximity at the middle of the screen. A CSPC effect was found for outer but not middle mostly compatible and mostly incompatible locations, suggesting the attentional setting bound to the middle locations reflected the average history of conflict (i.e., $50 \%$ compatible) for the group of middle locations. In Experiment 2, distance between middle locations was increased, allowing middle locations to group with outer locations. The CSPC effect was found for outer and middle mostly compatible and mostly incompatible locations. Results support the relative proximity hypothesis, positing that attentional settings bound to a particular location are
\end{abstract}

Nathaniel T. Diede

ndiede@wustl.edu

1 Washington University in St. Louis, St. Louis, MO, USA influenced by experience within a location and relatively close neighboring locations.

Keywords Context-specific cognitive control · Proportion congruence $\cdot$ Flanker $\cdot$ Spatial proximity

Imagine you are typing away at an important paper. Suddenly, an alert pops up from the bottom right corner of your screen: "Upgrade your computer's protection for only \$19.99 per month!" You duly ignore it and get back to your work. Later, a new message pops up in the same corner: "New updates are available!" You cannot be bothered now, and return to your work. After a particularly productive stretch of writing, you stop to check your e-mail. You see that a new message was received, which is strange to you - normally, a popup alert in the bottom right corner informs you of new messages. Did it not pop-up, or did you just miss it?

The ability to maintain goal-directed behavior (e.g.,writing a paper) in the face of conflicting response tendencies (e.g., gazing at pop-ups) is facilitated by various cognitive control processes. In laboratory settings, one way these processes are investigated is by using the flanker task (Eriksen \& Eriksen, 1974). Participants view strings of arrows and select the response that corresponds to the direction indicated by the central arrow. On compatible trials, the flanking arrows do not conflict with the central arrow (e.g., $<<<<<<<$ ). However, on incompatible trials, the flanking arrows cue a response that conflicts with the central arrow (e.g., $<<<><<<$ ), slowing down responding. The difference in response time between compatible and incompatible trials is referred to as the compatibility effect, which indicates the efficiency of attentional selection.

One approach to examining cognitive control in the flanker task is to manipulate the proportion of compatible (PC) stimuli. In task conditions associated with a history of conflict 
(e.g., mostly incompatible items or lists), the compatibility effect is reduced compared to conditions not associated with a history of conflict (e.g., mostly compatible items or lists; for item-level PC effects, see Bugg, 2015; Wendt \& LunaRodriguez, 2009; for list level, see Hommel, 1994; Mattler, 2006). Of relevance to the current study is the context-specific proportion compatibility (CSPC) manipulation (Crump, Gong, \& Milliken, 2006), which varies the PC of distinct contexts, such as locations on-screen. For example, an upper location may be mostly compatible (i.e., on most trials all arrows point in the same direction), while a lower location may be mostly incompatible (i.e., on most trials the central and flanking arrows point in different directions), such that each location is associated with a different history of conflict. As a result, the compatibility effect in the mostly compatible location is larger than that in the mostly incompatible location - an effect called the CSPC effect (Bugg, 2014; Corballis \& Gratton, 2003; Crump et al., 2006; Crump \& Milliken, 2009; Crump, Vaquero, \& Milliken, 2008; King, Korb, \& Egner, 2012; Vietze \& Wendt, 2009; Wendt, Kluwe, \& Vietze, 2008; Zurawska vel Grajewska, Sim, Hoenig, Herrnberger, \& Kiefer, 2011). The CSPC effect demonstrates a fast and flexible form of cognitive control, hereafter referred to as context-specific control, which reactively adapts attention in response to contextual cues. These adaptations are assumed to occur "on the fly" because participants cannot predict on any given trial in which context the next stimulus will appear, preventing a proactive shift of attention for an upcoming trial (see Crump et al., 2006, for evidence that the CSPC effect does not reflect awareness of the proportion compatibility manipulation).

An outstanding issue yet to be addressed is what causes a given contextual cue (location) to be associated with a particular adaptation of attention (i.e., attentional setting). The Gestalt principle of spatial proximity refers to the idea that visual stimuli that are closer to one another are grouped together (Pomerantz \& Portillo, 2011). Inspired by this principle, the aim of the current study was to examine if the relative spatial proximity of particular locations influences how these locations are grouped in regions (e.g., upper vs. middle vs. lower) of visual space, and consequentially, the binding of these locations to particular attentional settings (hereafter referred to as the relative proximity hypothesis). For instance, it may be that a mostly incompatible location and a mostly compatible location within relatively close proximity to one another tend to be grouped, leading to an absence of the typical CSPC effect that is observed when comparing compatibility effects across these two locations. Conversely, breaking up such a grouping may lead to a "reemergence" of the CSPC effect. Investigating this issue not only may extend Gestalt principles to the domain of cognitive control (cf., Luo \& Proctor, 2016), and to a paradigm in which stimuli never appear concurrently, but it also offers a fruitful avenue for exploring a prominent account of CSPC effects, the episodic retrieval account, which we introduce next.

\section{Episodic Retrieval Account of CSPC Effects}

The CSPC effect may be explained by the formation of stimulus-attention representations that are retrieved automatically when cued by a context (see Bugg \& Crump, 2012, for a review). The retrieval of episodic representations was most clearly demonstrated by Crump and Milliken (2009), who found that attentional settings generalized to unbiased stimuli that appeared in contexts associated with different histories of conflict. In a modified Stroop paradigm, a color word was initially presented (in white font) immediately followed by a color patch that the participant would then name. The locations the color patches could appear in were associated with two different histories of conflict. These histories were induced by a set of biased items (e.g., green and white color patches that followed the words GREEN or WHITE) that were either mostly compatible or mostly incompatible, depending on the location the color patch appeared. Most importantly, two unique items (e.g., blue and yellow patches that followed the words BLUE or YELLOW) were unbiased, meaning they had a $50 \%$ chance of being compatible or incompatible regardless of the location the color patch was presented. Both biased and unbiased items exhibited a CSPC effect (i.e., a larger compatibility effect when a color patch was presented in the mostly compatible location). The finding of a CSPC effect for unbiased stimuli demonstrates that the attentional settings underlying context-specific control are sufficiently abstract to handle stimulus variability, refuting a complex stimulus-response (associative learning) account of the CSPC effect (cf. Schmidt \& Besner, 2008). Instead, Crump and Milliken proposed an episodic retrieval mechanism to explain these results. The episodic retrieval account posits that context cues are bound with the attentional settings that have been used when responding to stimuli within a context, forming an episodic representation. When a stimulus appears in a given context, the episodic representation is rapidly retrieved along with the associated attentional settings.

The episodic retrieval account assumes that a given contextual cue (e.g., upper location) becomes bound with the attentional setting that has been used when responding to stimuli in that location in the past. In the CSPC paradigm, such bindings would lead to a broad attentional setting at the mostly compatible location (i.e., in a flanker task, a distribution of attention across target and flanker arrows) and a narrow attentional setting at the mostly incompatible location (i.e., reduced processing of flanker arrows). One possibility is that the attentional setting that is bound to a given location reflects prior experiences (with stimuli) in that particular location, as a strong location-specific interpretation of the episodic retrieval 
account would suggest. However, it is also possible that the attentional setting that is bound to a given location reflects experiences with stimuli in multiple locations or a region of space. For instance, if two locations of opposing biases (mostly compatible vs. mostly incompatible) were placed within close spatial proximity to one another relative to other locations, the two locations may be represented in a single episode to which an attentional setting is bound that corresponds (approximately) to the average PC (i.e., $50 \%$ compatible) of the two locations. Accordingly, both the mostly compatible and mostly incompatible locations would be expected to trigger a similar attentional setting such that a CSPC effect would not be found.

Although Gestalt principles typically refer to lower level perceptual phenomena related to statically and concurrently presented objects, spatial proximity has been shown to influence feature integration of temporally separated stimuli. For instance, the remaining percept of rapidly presented lines with oppositely offset segments has been shown to reflect the average of the two offset segments (with a slight bias to the most recent segment; Hermens, Scharnowski, \& Herzog, 2009). It

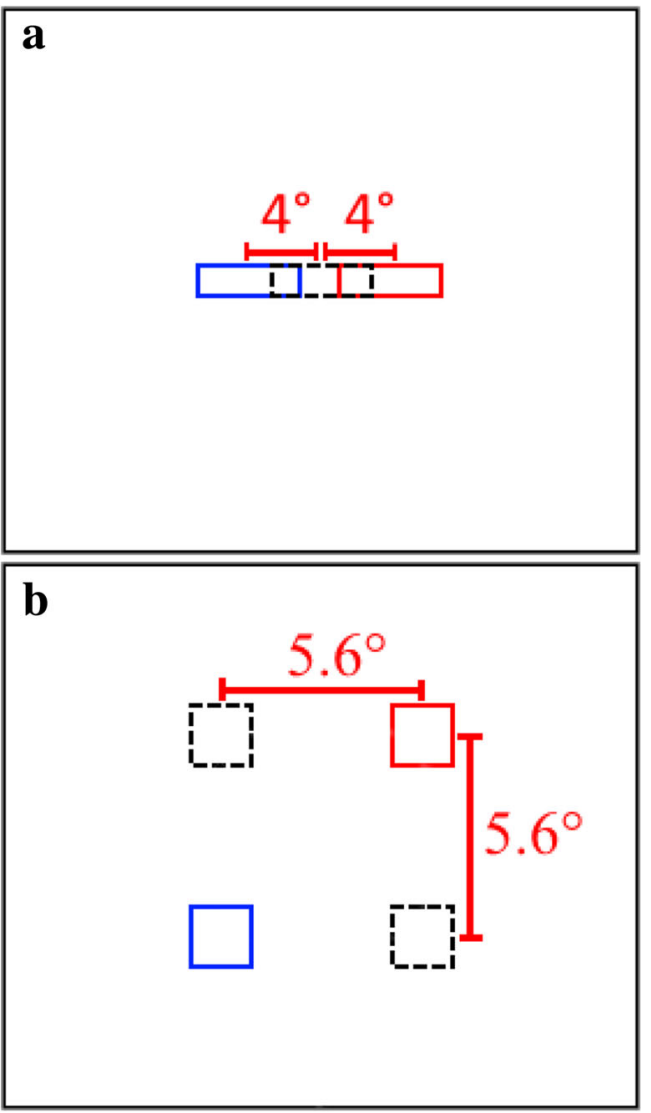

Fig. 1 Schematic depiction of the relative locations in degrees of visual angle used in (a) Corballis and Gratton (2003) and (b) Wendt, Kluwe, and Vietze (2008). Blue squares denote mostly incompatible locations, red squares denote mostly compatible, and dashed lines denote unbiased locations. (Color figure online) is not yet known if a similar mechanism might operate within a CSPC paradigm, influencing higher level cognitive control processes, but finding so would substantially inform the episodic retrieval account and extend the known influence of the principle of spatial proximity.

A strong test of the relative proximity hypothesis requires a paradigm that utilizes more than two locations in order to allow for the "grouping" of stimulus locations. Two prior CSPC studies have used paradigms with more than two locations, but these studies have not been able to clearly ascertain the effect of relative spatial proximity on context-specific control. Corballis and Gratton (2003; Experiment 1) placed an unbiased (50\% compatible) location at the center of a screen with a mostly incompatible location on the left and a mostly compatible location on the right (see Fig. 1A). A CSPC effect was found when comparing the mostly incompatible location to the mostly compatible location, while the compatibility effect for the unbiased location was intermediate. These results could be explained by the relative proximity hypothesis, in that the attentional setting bound to the middle location could reflect an average of the two settings that were bound to the surrounding and equally distant mostly incompatible and mostly compatible locations. However, a strong location-specific version of the episodic retrieval account could also explain the effect, whereby a given location is bound with an attentional setting that corresponds to the PC of that specific location. ${ }^{1}$ Similarly, a study by Wendt et al. (2008) could also be interpreted in either way. Four locations were presented equidistantly, landing on the points of an imaginary square at the middle of a computer screen (see Fig. 1B). The mostly incompatible and mostly compatible locations were diagonal from one another (e.g., bottom-left mostly incompatible; top-right mostly compatible), while the remaining two locations were unbiased (e.g., top-left and bottom-right locations were $50 \%$ compatible). Similar to Corballis and Gratton, the two unbiased locations both had similar, moderate compatibility effects, while the mostly incompatible location showed the smallest and the mostly compatible location the largest (i.e., a CSPC effect). Again, the moderate effects for the unbiased locations could be explained

\footnotetext{
${ }^{1}$ In Experiment 3 of Corballis and Gratton (2003), there were two lateral locations and one central location. The compatibility effect in the central, $50 \%$ location varied depending on the PC of the lateral locations. Critically, the lateral locations were always associated with the same PC bias (mostly compatible or mostly incompatible, which differed across blocks of trials) such that the overall PC of the mostly compatible blocks was $67 \%$ whereas in the mostly incompatible blocks it was $33 \%$. Thus, it is possible that participants adopted a global control setting that was more stringent in the mostly incompatible than mostly compatible blocks for all stimuli, regardless of location. This global setting can account for the difference in compatibility effects in the central location across blocks. In the current experiments, the block-wise PC was $50 \%$, such that any differences in compatibility effects among locations could be unambiguously attributed to location-specific attentional sets, and not a global set.
} 
by the strong location-specific version of the episodic retrieval account, or by the equal relative proximity to the biased locations. For example, the top-left unbiased location's effect may be the result of the upper portion of the screen being encoded as mostly compatible, combined with the left portion of the screen being encoded as mostly incompatible, and the two contributions averaging to an intermediate PC and a corresponding attentional setting.

In Experiment 1, we modified the CSPC paradigm to provide a clearer test of the relative proximity hypothesis. As shown in Fig. 2B, like prior CSPC paradigms, an outer mostly compatible location was presented above fixation and an outer mostly incompatible location was presented below fixation (assignment of PC to location was counterbalanced). However, unlike prior paradigms, two additional mostly compatible locations (referred to hereafter as middle mostly compatible locations) were presented above fixation, albeit not in close proximity to the outer mostly compatible location. The same was true for two additional mostly incompatible locations (middle mostly incompatible locations), which were presented below fixation. Importantly, this yielded locations that differed in relative proximity - the two middle mostly compatible locations were closer to the two middle mostly incompatible locations (and vice versa) than they were to the more distal outer locations that shared their bias. If, consistent with the strong (location-specific) version of the episodic retrieval account, each location independently cues an associated attentional setting, then the compatibility effect for each location should reflect the PC of that location regardless of the PC of nearby locations. In other words, a CSPC effect should be evident not only when comparing the outer locations but also when comparing the middle mostly compatible locations to the middle mostly incompatible locations. However, if experiences in locations that are relatively proximal to one another contribute to the same episodic representation (and the attentional setting that is then bound to this representation), then the compatibility effect in the middle mostly compatible locations should be equivalent to that of the middle mostly incompatible locations (i.e., no CSPC effect). The outer locations, in contrast, should still exhibit a CSPC effect because they are relatively distal from the influence of the middle locations. This pattern of effects would support the relative proximity hypothesis, while refuting a strong location-specific episodic retrieval account, because it would demonstrate that the attentional setting associated with two locations of opposing biases is similar, reflecting approximately the average of the individual location PCs (biases). To preview, we observed this pattern of effects in Experiment 1, supporting the relative proximity hypothesis. Accordingly, in Experiment 2, the aim was to examine whether moving the middle locations closer to the outer locations that shared their particular biases would eliminate the middle episodic representation. If such "regrouping" occurs, then a CSPC effect should be found for the middle locations.

Finally, Experiments 1 and 2 also aimed to address a second question concerning the CSPC effect. In one of the initial reports of a CSPC effect, Crump et al. (2006) examined both location and shapes as contextual cues for control. Although they found that location successfully cued control, shape did not (i.e., the compatibility effect was equivalent for mostly compatible shapes and mostly incompatible shapes; see Crump et al., 2008, for conditions under which shape is an effective cue). They conjectured that location-based CSPC effects may benefit from "spatial uncertainty," a property inherent of location cues but not centrally presented shape cues. We tested the effect of spatial uncertainty on the magnitude of the CSPC effect by including a second condition where only the two outer locations were presented on-screen (see Fig. 2A). If spatial uncertainty facilitates learning about context-specific proportion congruence or the process by which attentional settings become bound to different locations, a larger CSPC effect should be found in the condition with six biased stimulus locations (the multiple location condition; Fig. 2B) compared to just two locations (the single location condition; Fig. 2A).

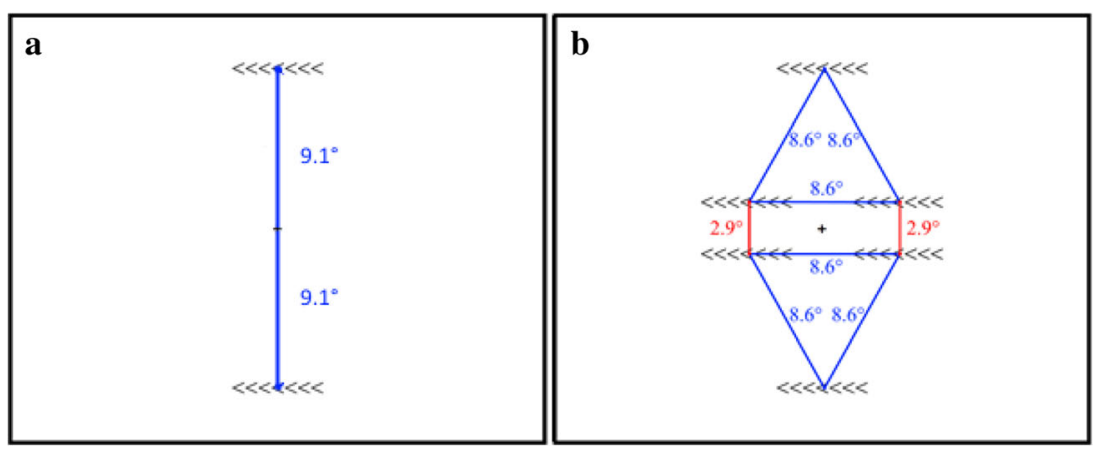

Fig. 2 Relative distances of stimulus locations in the single location condition (a) and the multiple location condition (b) in Experiment 1 . Note that only one flanker stimulus was presented per trial in one of the two (single location condition) or six (multiple location condition) possible locations 


\section{Experiment 1}

\section{Method}

Participants A sample of 48 participants was recruited from the Washington University student population (33 female, $\left.M_{\text {age }}=20.13, S D_{\text {age }}=1.67\right)$. Inclusion criteria were: age 18 to 25 years, right-handed, and self-reported normal or corrected to normal vision. All participants were compensated for their time by receiving either $\$ 5$ or partial course credit.

Stimuli The flanker stimuli were 5.6 degrees wide by 0.7 degrees tall, and comprised seven up, down, left, and/or right arrows. The central fixation cross was 0.5 degrees wide by 0.5 degrees tall. Flanker stimuli in the single location condition appeared 9.1 degrees above and below the central fixation cross (see Fig. 2A). The two locations used in the single location condition were also used in the multiple location condition, hereafter termed the outer locations. In addition, the multiple location condition included two middle locations in both the upper and lower halves. The middle locations were located 4.5 degrees from the central fixation cross, 8.6 degrees from all other stimuli in a given half of the screen, and 2.9 degrees from the nearest location in the opposite half of the screen (see Fig. 2B). The middle locations were chosen in order to form equilateral triangles when measured from target arrow to target arrow between the three locations presented in a given half. This allowed for broad coverage of a given half of the screen (in order to imply a top vs. bottom context) while still having a reasonable number of trials per location for statistical analysis.

Design A $2 \times 2 \times 2$ mixed design was used with trial type (compatible vs. incompatible) and PC (mostly compatible vs. mostly incompatible) as within-subjects factors and number of locations (NOL: single location vs. multiple location) as the between-subjects factor. Trial type referred to whether a stimulus was compatible (all arrows pointing in the same direction) or incompatible (central target arrow facing a different direction than the flanking arrows). There were four possible compatible stimuli (e.g., all arrows pointing right comprised one possibility) and 12 possible incompatible stimuli (e.g., a right-facing central arrow surrounded entirely by up, down, or left arrows comprised three such possibilities). PC was manipulated by varying the relative frequencies of compatible and incompatible trials that appeared on the top and bottom halves of the screen. For all locations in the mostly compatible half, $75 \%$ of trials were compatible whereas in the mostly incompatible half, all locations were $25 \%$ compatible. The PC of each half of the screen (mostly compatible or mostly incompatible) was counterbalanced across participants. Participants were randomly assigned to one of the two NOL conditions. The NOL conditions differed in the number of locations presented in a given half of the screen during experimental blocks. The single location condition presented only one location above fixation and one location below fixation (see panel A of Fig. 2). The multiple location condition presented three locations above and three below fixation (see panel B of Fig. 2).

Procedure Testing was conducted in a small room with a participant seated approximately $70 \mathrm{~cm}$ in front of a keyboard and a $1280 \times 1024$ LCD monitor running E-Prime. An experimenter sat to the participant's right, initially gaining consent and administering a demographics questionnaire. Participants were instructed to respond to the central arrow of the sevenarrow stimulus display by using their right index finger to press the corresponding button on a keyboard number pad (e.g., press the up [8], right [6], left [4], or down [2] key in response to an up, right, left, or down-facing central arrow, respectively), resting their finger on the 5 key after each response. The keys were covered with white labels. Instructions emphasized responding as quickly and accurately as possible. Twelve randomly presented practice trials were centrally presented, followed by three experimental blocks with brief breaks allowed in between.

All participants completed three blocks of 96 randomly presented trials. Within each block in the single location condition, 48 trials were presented in both the upper and lower halves of the screen. The same was true for the multiple location condition, albeit the 48 trials in each half were evenly distributed across the three locations in each half (i.e., the one outer and two middle locations). Stimuli were presented on-screen until a response, after which a fixation cross was presented for 1,000 ms (see Fig. 3). Reaction time and error rate were recorded. Once the task was complete, the experimenter provided the participant with a post-experiment questionnaire ${ }^{2}$ and debriefed them. The experiment lasted approximately 30 minutes.

\section{Results}

Trials on which reaction time (RT) was less than $200 \mathrm{~ms}$ or greater than 2,000 ms were eliminated from all analyses, excluding less than $.01 \%$ of trials (Bugg, 2015; Weidler \& Bugg, 2016). Additionally, trials on which an error was committed were excluded from RT analyses. On average, errors were made on less than $2 \%$ of trials. In this and the subsequent experiment, an alpha level of .05 was set with partial eta squared used as the measure of effect size. Besides those effects reported, no other effects were significant. Because error rates were overall low

\footnotetext{
${ }^{2}$ The post experiment questionnaire asked about awareness of the PC manipulation and replicated previous findings in the literature demonstrating that participants are poor at estimating the $\mathrm{PC}$ of the upper and lower locations (Crump, et al., 2006; Crump, et al., 2008). The results are thus not reported here.
} 
$\mathbf{a}$

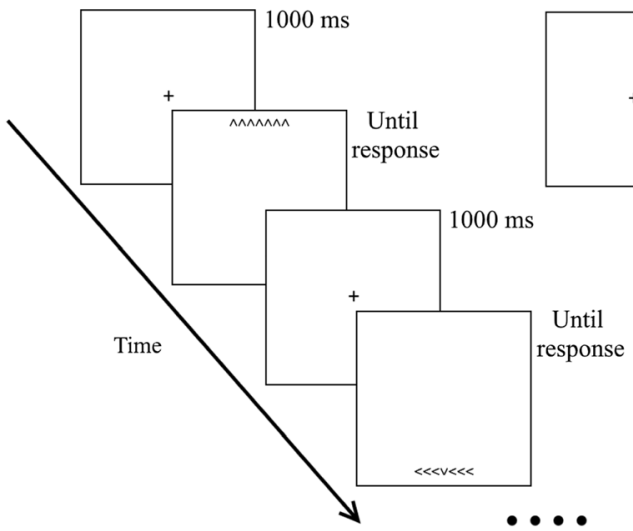

b

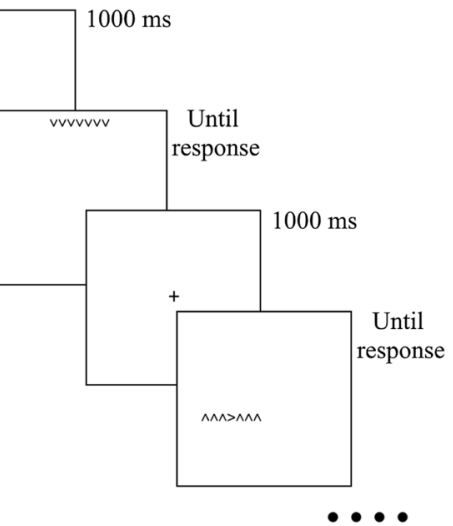

Fig. 3 Timeline representing progression of two trials in the (a) single and (b) multiple locations conditions. Stimuli not drawn to scale

and did not contradict the RT analyses, they are not reported (see Table 1 for means).

Descriptive statistics for all RT analyses are presented in Table 2. First, to test if a CSPC effect was present for outer locations, which would serve as a replication (single location condition) and extension (multiple location condition) of prior studies, a 2 (Trial Type) $\times 2(\mathrm{PC}) \times 2(\mathrm{NOL})$ mixed-design ANOVA was conducted on the outer locations. A significant Trial Type $\times \mathrm{PC}$ interaction was found, $F(1,46)=11.83$, MSE $=472, p=.001, \eta_{\mathrm{p}}^{2}=.205$, due to a CSPC effect of $22 \mathrm{~ms}$. The CSPC effect did not vary across the single location and multiple locations conditions, as indicated by the nonsignificant Trial Type $\times \mathrm{PC} \times$ NOL interaction, $F<1$. A main effect of Trial Type, $F(1,46)=489.66, M S E=1,736, p<.001$, $\eta_{\mathrm{p}}^{2}=.914$, indicated that RT was slower on incompatible trials $(M=798 \mathrm{~ms})$ compared to compatible trials ( $M=665 \mathrm{~ms})$.

Next, to test the critical prediction of the relative proximity hypothesis concerning the potential contribution of one's experience in proximal locations to the creation of a single episodic representation, a 2 (Trial Type) $\times 2$ (PC) within-subjects ANOVA was performed on the middle locations within the multiple location condition. The Trial Type $\times$ PC interaction was not significant, $F<1$, indicating that the magnitude of the compatibility effect was equivalent for the middle mostly compatible and middle mostly incompatible locations (i.e., there was not a CSPC effect; see Fig. 4). Only the main effect of trial type was significant, $F(1,23)=443.96, M S E=1,638$, $p<.001, \eta_{\mathrm{p}}^{2}=.951$, due to slower RT on incompatible trials ( $M=797 \mathrm{~ms})$ compared to compatible trials $(M=622 \mathrm{~ms})$.

A final aim was to test the secondary question that increasing spatial uncertainty will influence the magnitude of the CSPC effect, which can be addressed by comparing the single location and multiple location conditions. Previously it was reported that when comparing only the outer locations across conditions, there was no Trial Type $\times \mathrm{PC} \times \mathrm{NOL}$ interaction, indicating that the CSPC effect was not affected by spatial uncertainty. An alternative approach to examining this question is to submit mean RTs collapsed across all locations within a given half of the screen to a 2 (Trial Type: compatible vs. incompatible $) \times 2$ (PC: mostly compatible vs. mostly incompatible) $\times 2$ (NOL: single location vs. multiple location) mixed-design ANOVA. Although a Trial Type $\times$ PC interaction was found, $F(1,46)=8.53, M S E=324, p=.005$, $\eta_{\mathrm{p}}^{2}=.156$, due to a $16 \mathrm{~ms}$ CSPC effect, the Trial Type $\times$ $\mathrm{PC} \times \mathrm{NOL}$ interaction was not significant, $F<1$.

Table 1 Mean Error Rates and Standard Deviations for Experiments 1 and 2

\begin{tabular}{|c|c|c|c|c|c|c|c|c|c|}
\hline \multirow[t]{3}{*}{ Experiment } & \multirow[t]{3}{*}{ Stimuli Locations } & \multicolumn{4}{|c|}{ Single Location Condition } & \multicolumn{4}{|c|}{ Multiple Location Condition } \\
\hline & & \multicolumn{2}{|c|}{ Mostly Compatible } & \multicolumn{2}{|c|}{ Mostly Incompatible } & \multicolumn{2}{|c|}{ Mostly Compatible } & \multicolumn{2}{|c|}{ Mostly Incompatible } \\
\hline & & Compatible & Incompatible & Compatible & Incompatible & Compatible & Incompatible & Compatible & Incompatible \\
\hline \multirow[t]{2}{*}{1} & Outer Locations & $0.1(0.01)$ & $2.4(0.1)$ & $0.0(0.01)$ & $1.1(0.06)$ & $0.0(0.01)$ & $0.04(0.05)$ & $0.0(0.02)$ & $0.03(0.05)$ \\
\hline & Middle Locations & - & - & - & - & $0.0(0.00)$ & $0.05(0.06)$ & $0.0(0.01)$ & $0.04(0.04)$ \\
\hline \multirow[t]{2}{*}{2} & Outer Locations & $0.4(0.01)$ & $3.3(0.09)$ & $0.0(0.01)$ & $2.6(0.07)$ & $0.0(0.01)$ & $0.02(0.04)$ & $0.0(0.0)$ & $0.03(0.04)$ \\
\hline & Middle Locations & - & - & - & - & $0.0(0.00)$ & $0.05(0.05)$ & $0.0(0.01)$ & $0.04(0.05)$ \\
\hline
\end{tabular}

Note:Values outside of parentheses are means, those within are standard deviations. A "_" means error rate could not be calculated for this cell because there were no middle locations in the single location condition. 
Table 2 Mean Reaction Times and Standard Deviations for Experiments 1 and 2

\begin{tabular}{|c|c|c|c|c|c|c|c|c|c|}
\hline \multirow[t]{3}{*}{ Experiment } & \multirow[t]{3}{*}{ Stimuli Locations } & \multicolumn{4}{|c|}{ Single Location Condition } & \multicolumn{4}{|c|}{ Multiple Location Condition } \\
\hline & & \multicolumn{2}{|c|}{ Mostly Compatible } & \multicolumn{2}{|c|}{ Mostly Incompatible } & \multicolumn{2}{|c|}{ Mostly Compatible } & \multicolumn{2}{|c|}{ Mostly Incompatible } \\
\hline & & Compatible & Incompatible & Compatible & Incompatible & Compatible & Incompatible & Compatible & Incompatible \\
\hline \multirow[t]{2}{*}{1} & Outer Locations & $655(96)$ & $800(101)$ & $663(102)$ & $790(108)$ & $662(74)$ & $804(83)$ & $679(78)$ & $796(64)$ \\
\hline & Middle Locations & - & - & - & - & $622(74)$ & $800(79)$ & $623(68)$ & $793(61)$ \\
\hline \multirow[t]{2}{*}{2} & Outer Locations & $614(59)$ & $761(65)$ & $623(64)$ & $746(67)$ & $644(68)$ & $809(111)$ & $668(66)$ & $794(69)$ \\
\hline & Middle Locations & - & - & - & - & $617(65)$ & $793(87)$ & $629(66)$ & $787(75)$ \\
\hline
\end{tabular}

Note:Values outside of parentheses are means, those within are standard deviations. A “-” means reaction time could not be calculated for this cell because there were no middle locations in the single location condition.

Converging with the analysis of outer locations, this indicates that CSPC effects were similar in magnitude in the single location $(M=18 \mathrm{~ms})$ and multiple location conditions ( $M=13 \mathrm{~ms}$; see Fig. 4). For completeness, it is noted that a main effect of trial type, $F(1,46)=746.52, M S E=$ $1405, \mathrm{p}<.001, \eta_{\mathrm{p}}^{2}=.942$, was found, due to slower RT on incompatible $(M=797 \mathrm{~ms})$ compared to compatible trials $(M=649 \mathrm{~ms})$, as was a Trial Type $\times$ NOL interaction, $F(1,46)=4.38, M S E=1405, p=.042, \eta_{\mathrm{p}}^{2}=.087$. This was due to a larger compatibility effect in the multiple location condition $(M=159 \mathrm{~ms})$ compared to the single location condition $(M=136 \mathrm{~ms})$.

\section{Discussion}

The primary question examined in Experiment 1 was whether a CSPC effect would be found for locations of opposing biases (mostly compatible vs. mostly incompatible) under conditions that encouraged the grouping of these locations (i.e., they were

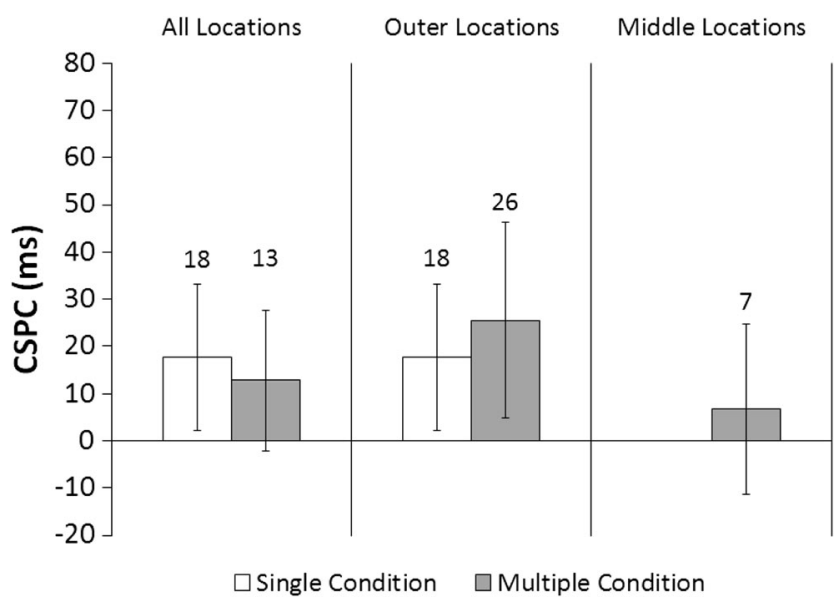

Fig. 4 Average context-specific proportion congruency (CSPC) effects in Experiment 1. Outer locations are the uppermost and lowermost locations that are present in both single location and multiple location conditions. Middle locations are present only in the multiple location condition. Error bars represent $95 \%$ confidence intervals. placed closer to one another relative to other locations that shared their biases). A strong location-specific version of the episodic retrieval account predicted that a CSPC effect would be present when comparing the middle mostly compatible locations to the middle mostly incompatible locations of the multiple location condition. In contrast, the relative proximity hypothesis allowed for the possibility that the two proximal but opposing locations may be grouped together, such that experiences within these locations would contribute to the same episodic representation. In particular, the average of the two PCs (biases) would result in an unbiased, $50 \%$ compatible representation (and accordingly, an intermediate attentional setting), and therefore no CSPC effect. Supporting the relative proximity hypothesis, there was not a CSPC effect for the middle locations. In contrast, mirroring prior studies (Corballis \& Gratton, 2003; Crump et al., 2006; Crump \& Milliken, 2009; Crump et al., 2008; King et al., 2012; Vietze \& Wendt, 2009; Wendt et al., 2008; Zurawska vel Grajewska et al., 2011), a CSPC effect was found for the outer mostly compatible and mostly incompatible locations (in both the multiple location and single location conditions). The findings for the multiple location condition therefore suggest that three episodic representations were formed: one mostly compatible representation and one mostly incompatible representation, which produced the outer location CSPC effect, and one large unbiased representation formed by the four middle locations.

The second question examined in Experiment 1 was whether increasing the number of biased locations influences the magnitude of the CSPC effect. When comparing all locations in the upper half of the screen to those in the lower half of the screen, no difference in the CSPC effect was found between the single location and multiple location condition. One possibility is that this result was influenced by the formation of the middle unbiased representation in the multiple location condition, thereby weakening any effect multiple locations may have had on strengthening the CSPC effect. However, no difference in the CSPC effect was found when comparing only the outer locations within the single location and multiple location conditions (though the CSPC effect in the multiple 
location condition was numerically higher). These findings suggest that, contrary to the speculation of Crump et al. (2006), purely having less certainty of where the next stimulus will appear does not seem to influence the strength of the CSPC effect.

\section{Experiment 2}

Experiment 1 demonstrated for the first time that relative spatial proximity matters when determining what attentional setting is bound to a particular location in a CSPC paradigm. When locations of two opposing biases (mostly compatible vs. mostly incompatible) were placed relatively close to one another, they functioned as if they were associated with a single episodic representation reflecting (approximately) the average PC of the two locations (50\% compatible). ${ }^{3}$ That is, presentation of a stimulus in any of the middle locations appeared to stimulate retrieval of a similar attentional setting such that the magnitude of compatibility effects did not differ between mostly compatible and mostly incompatible middle locations.

Experiment 2 tested for the inverse pattern needed to demonstrate the role of spatial proximity in the creation of episodic representations in CSPC paradigms - if the relative distance between opposing biased locations (mostly compatible and mostly incompatible) is increased, a CSPC effect should emerge when contrasting the compatibility effects across these locations. Accordingly, the middle locations were moved farther apart while the distance between biased locations (e.g., the mostly compatible locations) on a given half of the screen was decreased (see Fig. 5). Following from the Gestalt principle of spatial proximity, it was expected that the middle locations would no longer group; instead, a new (biased) group was expected to form among the middle and outer locations on a given half of the screen. Based on the relative proximity hypothesis, it was predicted that two episodic representations would be formed, one for the three mostly compatible locations and one for the three mostly incompatible locations, thus producing a significant CSPC effect for the middle (and outer) locations.

Additionally, the role of increased spatial uncertainty was again tested. Experiment 1 failed to find an increase in the CSPC effect in the multiple location condition, possibly due

\footnotetext{
${ }^{3}$ Although the suggestion is that the middle locations will group and the attentional setting will reflect the average of those locations (a PC of $50 \%$ compatible), we caution against comparing the magnitude of compatibility effects in middle locations to the outer mostly compatible and outer mostly incompatible locations as a test of the "intermediacy" of the attentional setting of the middle locations. This is because the distance the eyes travel when responding to middle versus outer locations confounds these comparisons, and can affect the magnitude of compatibility effects (see Corballis \& Gratton, 2003; Weidler \& Bugg, in press, for similar cautionary notes).
}

to the formation of the central unbiased representation. If reducing the relative spatial distance between locations of the same PC (bias) eliminates the middle unbiased representation, then a larger CSPC effect may be observed in the multiple location condition compared to the single location condition, consistent with the speculation of Crump et al. (2006).

\section{Method}

Participants. A sample of 49 participants was recruited by the same means and inclusion criteria as Experiment 1. A single participant was excluded for not following task instructions (responding to distractor arrows rather than the target arrow). The final sample size was 48 (33 female, $\left.M_{\text {age }}=19.08, S D_{\text {age }}=1.20\right)$. Participants received either $\$ 5$ or partial course credit.

Stimuli As in Experiment 1, the outer locations were 9.1 degrees above and below fixation; however, the middle locations were spread apart to a distance of 8.4 degrees from the nearest location in the opposite half of the screen. In order to maintain equal distance between stimulus locations in a given half, the distance between each location was reduced from 8.6 degrees to 5.6 degrees. This caused each middle location to then be 5 degrees from the fixation cross (see Fig. 5).

Procedure Experiment 2 proceeded exactly as Experiment 1, with only the relative distance of stimulus locations in the multiple location condition changed.

\section{Results}

The same RT outlier trimming procedures were applied as in Experiment 1, excluding less than $.01 \%$ of trials. Trials on which an error was committed were excluded from RT analyses. Overall error commission was again low $(M=2 \%)$ and reflected the same pattern as the RT analyses (see Table 1); therefore, error analyses are not reported.

Descriptive statistics for RT analyses are presented in Table 2. First, outer locations were examined to see if the typical CSPC effect was present in the single and multiple location conditions. As in Experiment 1, the 2 (Trial Type) $\times$ $2(\mathrm{PC}) \times 2(\mathrm{NOL})$ mixed design ANOVA found a significant Trial Type $\times$ PC interaction, $F(1,46)=20.01, M S E=596$, $p<.001, \eta_{\mathrm{p}}^{2}=.303$, due to a CSPC effect of $31 \mathrm{~ms}$, and this effect did not differ between the single and multiple location conditions $(F(1,46)=1.10, M S E=596, p=.300$, $\eta_{\mathrm{p}}^{2}=.023$, for the Trial Type $\times \mathrm{PC} \times \mathrm{NOL}$ interaction) . The main effect of Trial Type was also significant, $F(1,46)$ $=584.60, M S E=1,608, p<.001, \eta_{\mathrm{p}}^{2}=.927$, due to slower RT on incompatible trials ( $M=777 \mathrm{~ms}$ ) compared to compatible trials $(M=637 \mathrm{~ms})$. 


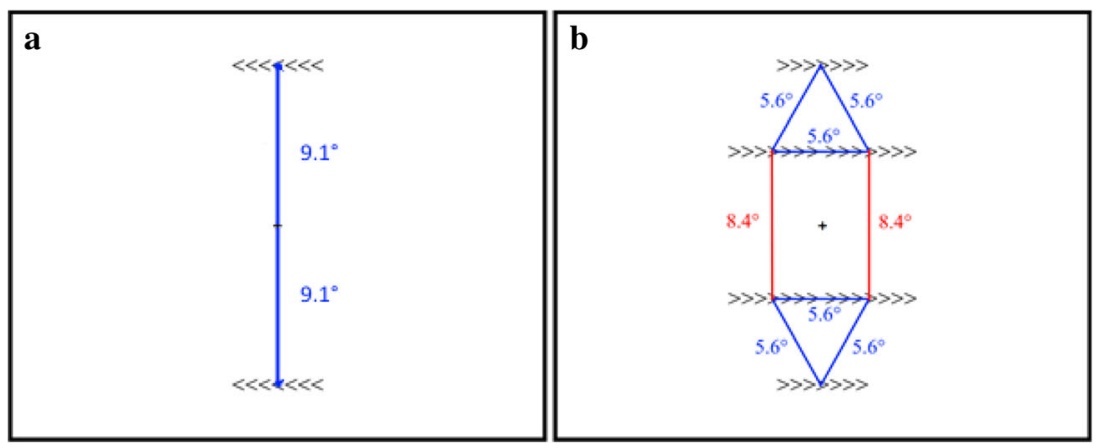

Fig. 5 Relative distances of stimulus locations in the single location condition (a) and the multiple location condition (b) in Experiment 2 . Note that only one flanker stimulus was presented per trial in one of the two (single location condition) or six (multiple location condition) possible locations.

Next, a 2 (Trial Type) $\times 2$ (PC) within-subjects ANOVA was conducted to determine if modifying relative proximity led to a CSPC effect for the middle locations in the multiple location condition. A significant Trial Type $\times \mathrm{PC}$ interaction was found, $F(1,23)=5.82, M S E=342, p=.024, \eta_{\mathrm{p}}^{2}=.202$, indicating a CSPC effect of $18 \mathrm{~ms}$ (see Fig. 6). ${ }^{4}$ The main effect of trial type was also significant, $F(1,23)=320.93$, $M S E=2,084, p<.001, \eta_{\mathrm{p}}^{2}=.933$, due to slower RT on incompatible trials $(M=790 \mathrm{~ms})$ compared to compatible trials $(M=623 \mathrm{~ms})$.

Finally, the magnitude of the CSPC effect was compared between the upper and lower halves of the screen (collapsed across locations within a given half) to further gauge the role of spatial uncertainty. As noted previously, the comparison of the outer locations revealed equivalent CSPC effects across the single and multiple locations conditions, inconsistent with the spatial uncertainty hypothesis. A 2 (Trial Type) $\times 2(\mathrm{PC}) \times$ 2 (NOL) mixed-design ANOVA revealed a significant Trial Type $\times$ PC interaction, $F(1,46)=23.39, M S E=311, p<.001$, $\eta_{\mathrm{p}}^{2}=.337$, due to a CSPC effect of $26 \mathrm{~ms}$. However, the Trial Type $\times \mathrm{PC} \times$ NOL interaction was again not significant, $F<1$, indicating equivalent CSPC effects for the single and multiple location conditions (see Fig. 6). For completeness, it is noted that there was a main effect of trial type, $F(1,46)=701.88, M S E=1,480, p<.001, \eta_{\mathrm{p}}^{2}$ $=.938$, due to slower $\mathrm{RT}$ on incompatible trials $(M=$ $773 \mathrm{~ms})$ compared to compatible trials $(M=626 \mathrm{~ms})$, and a significant Trial Type $\times$ NOL interaction, $F(1,46)$ $=5.27, M S E=1,480, p=.026, \eta_{\mathrm{p}}^{2}=.103$. This was due to a larger compatibility effect in the multiple location condition $(M=160 \mathrm{~ms})$ compared to the single location condition $(M=134 \mathrm{~ms})$.

\footnotetext{
${ }^{4}$ Numerically, the CSPC effect for the outer locations in Experiment 2 was larger than in Experiment 1 . This raises the possibility that the CSPC effect for the middle locations in Experiment 2 was due to a generally increased tendency for PC-based attentional adjustments in that experiment. To examine this possibility, we submitted RTs from the outer locations to a $2(\mathrm{PC}) \times 2$ (Trial Type) $\times 2$ (Experiment) mixed design ANOVA. Countering this possible explanation, no effects of Experiment were significant (all $p \mathrm{~s} \geq .23$; of greatest relevance, for the $\mathrm{PC} \times$ Trial Type $\times$ Experiment interaction, $F<1)$.
}

\section{Discussion}

Consistent with the relative proximity hypothesis, a CSPC effect emerged for middle locations when participants were encouraged to group the middle locations with the outer locations. This was accomplished by moving the middle locations apart. This suggests that reducing the relative distance between locations of the same bias (PC), and thereby increasing the relative distance between opposing biases, led to the formation of distinct episodic representations for each group of locations. One representation was formed for the grouped locations above fixation (e.g., in the mostly compatible half). A broader attentional setting was bound to that representation than the representation formed for the grouped locations below fixation (e.g., in the mostly incompatible half), as indicated by the larger compatibility effect in the mostly congruent locations compared to the mostly incongruent locations.

As in Experiment 1, increased spatial uncertainty did not seem to modulate the CSPC effect. No difference in the CSPC effect was found between the single location and multiple

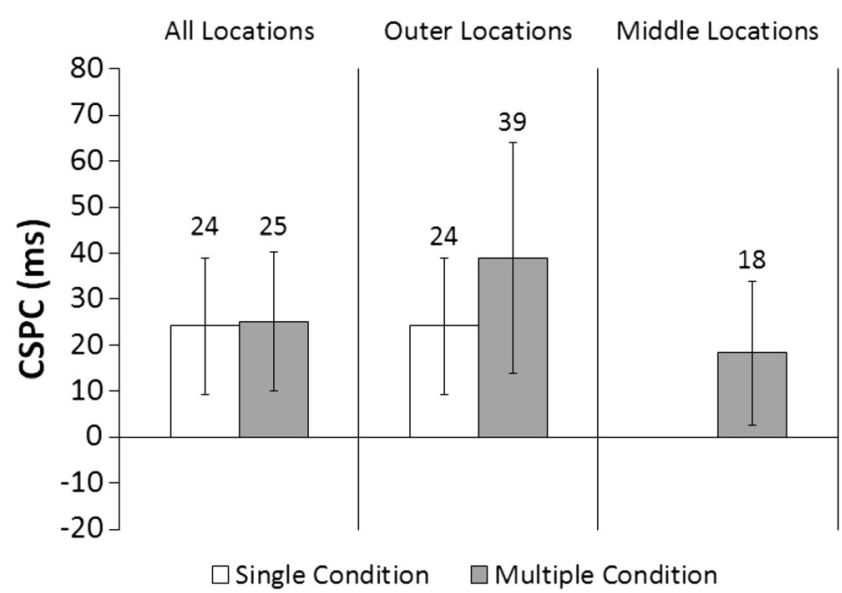

Fig. 6 Average context-specific proportion congruency (CSPC) effects in Experiment 2. Outer locations are the uppermost and lowermost locations that are present in both single location and multiple location conditions. Middle locations are present only in the multiple location condition. Error bars represent $95 \%$ confidence intervals 
location condition when comparing all locations in the upper versus the lower halves of the screen. The same was true when comparing CSPC effects in the outer locations of the single location and multiple location conditions, despite the multiple location condition again having a nominally larger CSPC effect.

\section{General Discussion}

In a series of two experiments examining CSPC effects, it was demonstrated that relative spatial proximity of locations influences the attentional setting bound to a given location. When locations of opposing histories (mostly compatible vs. mostly incompatible) were presented in close proximity, no CSPC effect was found. This suggests that each location triggered retrieval of an attentional setting that reflected the averaged histories of the two opposing but clustered locations. All the while, a CSPC effect was found for mostly compatible and mostly incompatible locations outside of the cluster (see Experiment 1). When locations with similar histories of conflict (e.g., mostly compatible locations) were presented closer to one another relative to locations of opposing histories (e.g., mostly incompatible locations), a CSPC effect was found between the two groups of locations (see Experiment 2). These findings appear to demonstrate the occurrence of a phenomenon reminiscent of the Gestalt principle of spatial proximity (Pomerantz \& Portillo, 2011). Gestalt principles have been shown to affect processes such as working memory (Peterson \& Berryhill, 2013; Woodman, Vecera, \& Luck, 2003) and attention (Beck \& Palmer, 2002) in paradigms where grouped visual stimuli were presented on-screen concurrently. In the current study, a single visual stimulus appeared at a given time. The current findings therefore suggest that perceptual grouping based on spatial proximity may occur even in the absence of concurrent presentation of stimuli (cf. Hermens et al., 2009), and such grouping may affect "higher level" cognitive processes, such as cognitive control of attention.

The current findings are not easily explained by a strong location-specific episodic retrieval account, according to which each location cues an independent episodic representation to which an associated attentional setting (based only on experience within a given location) has been bound. However, the current findings are consistent with the relative proximity hypothesis, and point to a fruitful expansion of the episodic retrieval account to explain what determines which experiences are bound together in an episodic representation. As with the account posited by Crump and Milliken (2009), experiences within a given location (context) are bound with an attentional setting. This attentional setting is automatically retrieved whenever that location is cued. However, the representation can be influenced by other locations of close relative spatial proximity, even when such locations are associated with a different $\mathrm{PC}$ level and therefore an alternative attentional setting. The current data suggest that the episodic representation is influenced approximately equally by the input at each location: if both locations present the same bias (mostly compatible or mostly incompatible), then the average bias of the representation is the same as the individual locations. Alternatively, if one location is $75 \%$ compatible (i.e., mostly compatible) and the other $75 \%$ incompatible (i.e., mostly incompatible), the influence of the two locations averages to an unbiased $50 \%$ compatible representation, washing out the CSPC effect between the two locations.

An alternative to the idea that the group takes on the average PC (and associated attentional setting) of the locations that have been grouped is that participants are simply unable to distinguish among the upper middle and lower middle locations, sometimes retrieving the incorrect control settings for a given location rather than retrieving a single shared episodic representation. Although we cannot rule out this explanation entirely, it seems somewhat improbable because a fixation cross appeared centrally during the inter-stimulus interval and provided a reference point for encoding the location of each stimulus (as occurring above or below fixation), and responding to the upper and lower middle locations entailed distinct eye movements (in four different directions). Future studies might aim to replicate the findings of Experiment 1 with a display that enhances the salience of (and potentially disrupts grouping of) the upper and lower middle locations, for instance, by placing a horizontal line in the middle of the screen or coloring each half of the screen a different color.

A secondary goal of the current experiments was to investigate the conjecture that spatial uncertainty may influence the magnitude of the CSPC effect (Crump et al., 2006). In neither experiment did the data statistically support this conjecture. However, the CSPC effect was nominally larger in the multiple location condition than in the single location condition in both experiments (but see Footnote 4). Future research is therefore needed to further test the spatial uncertainty hypothesis. For example, it may be that spatial uncertainty has an effect selectively in designs that contrast constant (as in colorbased manipulations of context) versus nonconstant locations (as in traditional location-based manipulations involving a single upper and lower location), or that an alternative arrangement of locations (e.g., having all locations of a given PC appear on the same horizontal line) may produce an effect.

Several limitations of the current study should be noted. First, an equivalent number of trials was presented in each location in the multiple location condition. As a result, more trials were presented at the middle of the screen compared to the outer edges of the screen in Experiment 1, such that trials in the outer locations were relatively more rare than trials in the middle locations. The implication is that outer locations were distinct from the middle locations not only due to relative 
spatial proximity, but also due to relative spatial novelty. This limitation is tempered by the results of Experiment 2, however, in which the outer locations were presented with the same relative rarity as Experiment 1, yet a CSPC effect was not limited to outer locations. Second, the stimuli used in the current study were wider than they were tall. Future studies may benefit from using flanker stimuli that are as tall as they are wide (such as those used by Wendt et al., 2008). Doing so would allow more precise control over the location at which a stimulus is presented.

Third, following on the finding that CSPC effects transfer to novel stimuli that are devoid of location-flanker response associations (see Crump \& Milliken, 2009), we have attributed the CSPC effects in the current study to location-specific attentional settings. However, it is possible that locationflanker-response association learning (or priming) may have contributed to the CSPC effect. In mostly compatible locations, rightward-facing flanking arrows (as an example) were more frequently presented with rightward-facing target arrows, which might have speeded responding on congruent trials, thereby increasing the size of the compatibility effect. Performance in the mostly incompatible locations, however, most likely reflected the application of a narrow attentional setting because a single response was not strongly associated with (or primed by) flanker arrows - rightward-facing flanker arrows (as an example) were presented equally frequently with all possible targets in the mostly incompatible locations. Accordingly, it is difficult to envision how an account of CSPC effects based on associative learning would explain the novel effects observed for the middle locations in the current experiments. Consider the Experiment 1 finding of no CSPC effect when comparing the mostly compatible and mostly incompatible middle locations. It is unclear how a learned response tendency (e.g., press right to right flankers) in the mostly compatible location would be averaged with an attentional setting (e.g., restrict attention to target) in the mostly incompatible location to produce this pattern. In contrast, this finding is readily explained by the averaging of two attentional settings (broader in mostly compatible and narrower in mostly incompatible).

Fourth, it is unclear whether relative spatial proximity influences the binding process solely in location-based CSPC paradigms. In recent years, other cues have been found to successfully signal context-specific proportion compatibility, such as shape (Crump et al., 2008), color (Heinemann, Kunde, \& Kiesel, 2009; Lehle \& Hübner, 2008; Vietze \& Wendt, 2009), and number parity (Reuss, Desender, Kiesel, \& Kunde, 2014). Future studies are therefore needed to determine whether other conceptualizations of proximity, such as proximity in categorical space, may influence binding in such CSPC paradigms. For instance, in a shape-specific paradigm, circular and oval contextual cues may more readily influence the same episodic representation than circular and square contextual cues. Considering the current findings from this perspective, relative spatial proximity may have allowed for the categorization of upper, middle, and lower categories of space (for which unique episodic representations were formed; see Experiment 1) or only upper and lower categories (see Experiment 2), depending on how locations clustered. In line with this idea, recent work has demonstrated that different attentional settings were bound to inner versus outer rings of a bulls-eye associated with different histories of conflict, such that CSPC effects transferred to novel unbiased locations within each ring, possibly because such locations fell within the same categories of space (Weidler \& Bugg, 2016). Similarly, gender category has previously been shown to both cue PC and affect whether the CSPC effect transfers to novel items (i.e., faces; Cañadas, Rodríguez-Bailón, Milliken, \& Lupiáñez, 2013), demonstrating that items closer in categorical space are grouped together. Interestingly, instructing participants to focus on individual characteristics of the faces disrupted categorization, and transfer to novel faces of the same gender no longer occurred (Cañadas et al., 2013).

\section{Conclusion}

In summary, the rapid modulations of cognitive control observed in CSPC paradigms sometimes reflect a more complex process than simply associating experience with a given location (of a certain PC bias) with a particular attentional setting. The novel, multiple location CSPC paradigms used in the current study demonstrate that experiences with other, nearby locations (including those of opposing biases) also appear to influence the attentional setting that is bound to a given location, thereby affecting the magnitude of CSPC effects. Accordingly, the current data suggest that, should we hope to detect potentially important alerts in that usually pesky bottom right corner of the computer screen, we should allow for a usually relevant set of alerts to appear in a nearby location.

Authors' Note Nathaniel T. Diede and Julie M. Bugg, Department of Psychological and Brain Sciences, Washington University in St. Louis.

These data were collected in partial fulfillment of N.T.D.'s master's degree, and portions of the data were reported at the 55th Annual Meeting of the Psychonomic Society, Long Beach, CA. We are grateful to Madeline Kleiner, Jason Li, Bridgette Shamleffer, and Jenny Weissman for assisting with data collection. N.T.D. was supported by an NIA Training Grant (T32AG000030-40) during the writing of this manuscript.

\section{References}

Beck, D. M., \& Palmer, S. E. (2002). Top-down influences on perceptual grouping. Journal of Experimental Psychology: Human Perception and Performance, 28(5), 1071-1084. doi:10.1037/0096-1523.28.5. 1071

Bugg, J. M. (2014). Evidence for the sparing of reactive control with age. Psychology and Aging, 29(1), 115-127. doi:10.1037/a0035270 
Bugg, J. M. (2015). The relative attractiveness of distractors and targets affects the coming and going of item-specific control: Evidence from flanker tasks. Attention, Perception, \& Psychophysics, 77(2), 373-389. doi:10.3758/s13414-014-0752-x

Bugg, J. M., \& Crump, M. J. C. (2012). In support of a distinction between voluntary and stimulus-driven control: A review of the literature on proportion congruence effects. Frontiers in Psychology, 3, 367. doi:10.3389/fpsyg.2012.00367

Cañadas, E., Rodríguez-Bailón, R., Milliken, B., \& Lupiáñez, J. (2013). Social categories as a context for the allocation of attentional control. Journal of Experimental Psychology: General, 142(3), 934-943. doi: $10.1037 / \mathrm{a} 0029794$

Corballis, P. M., \& Gratton, G. (2003). Independent control of processing strategies for different locations in the visual field. Biological Psychology, 64, 191-209. doi:10.1016/S0301-0511(03)00109-1

Crump, M. J. C., Gong, Z., \& Milliken, B. (2006). The context-specific proportion congruent Stroop effect: Location as a contextual cue. Psychonomic Bulletin \& Review, 13(2), 316-321.

Crump, M. J. C., \& Milliken, B. (2009). The flexibility of context-specific control: Evidence for context-driven generalization of item-specific control settings. The Quarterly Journal of Experimental Psychology, 62(8), 1523-1532. doi:10.1080/17470210902752096

Crump, M. J. C., Vaquero, J. M. M., \& Milliken, B. (2008). Contextspecific learning and control: The roles of awareness, task relevance, and relative salience. Consciousness and Cognition, 17, 22-36. doi: 10.1016/j.concog.2007.01.004

Eriksen, B. A., \& Eriksen, C. W. (1974). Effects of noise letters upon the identification of a target letter in a non-search task. Perception and Psychophysics, 16, 143-149.

Heinemann, A., Kunde, W., \& Kiesel, A. (2009). Context-specific primecongruency effects: On the role of conscious stimulus representations for cognitive control. Consciousness and Cognition, 18, 966976. doi:10.1016/j.concog.2009.08.009

Hermens, F., Scharnowski, F., \& Herzog, M. H. (2009). Spatial grouping determines temporal integration. Journal of Experimental Psychology: Human Perception and Performance, 35(3), 595610. doi:10.1037/a0013706

Hommel, B. (1994). Spontaneous decay of response code activation. Psychological Research, 56, 261-268.

King, J. A., Korb, F. M., \& Egner, T. (2012). Priming of control: Implicit contextual cuing of top-down attentional set. The Journal of Neuroscience, 32(24), 8192-8200. doi:10.1523/JNEUROSCI. 0934-12.2012

Lehle, C., \& Hübner, R. (2008). On-the-fly adaptation of selectivity in the flanker task. Psychonomic Bulletin \& Review, 15(4), 814-818. doi: 10.3758/PBR.15.4.814
Luo, C., \& Proctor, R. W. (2016). Perceptual grouping of objects occupied by target and flankers affects target-flanker interference. Attention, Perception, \& Psychophysics, 78, 251-362. doi:10. 3758/s13414-015-0986-2

Mattler, U. (2006). Distance and ratio effects in the flanker task are due to different mechanisms. Quarterly Journal of Experimental Psychology, 59, 1745-1763.

Peterson, D. J., \& Berryhill, M. E. (2013). The Gestalt principle of similarity benefits visual working memory. Psychonomic Bulletin \& Review, 20, 1282-1289. doi:10.3758/s13423-013-0460-x

Pomerantz, J. R., \& Portillo, M. C. (2011). Grouping and emergent features in vision: Toward a theory of basic Gestalts. Journal of Experimental Psychology: Human Perception and Performance, 37(5), 1331-1349. doi:10.1037/a0024330

Reuss, H., Desender, K., Kiesel, A., \& Kunde, W. (2014). Unconscious conflicts in unconscious contexts: The role of awareness and timing in flexible conflict adaptation. Journal of Experimental Psychology: General, 143(4), 1701-1718. doi:10.1037/a0036437

Schmidt, J. R., \& Besner, D. (2008). The Stroop effect: Why proportion congruent has nothing to do with congruency and everything to do with contingency. Journal of Experimental Psychology: Learning, Memory, and Cognition, 34(3), 514-523. doi:10.1037/0278-7393. 34.3.514

Vietze, I., \& Wendt, M. (2009). Context specificity of conflict frequencydependent control. The Quarterly Journal of Experimental Psychology, 62(7), 1391-1400. doi:10.1080/17470210802426908

Weidler, B. J., \& Bugg, J. M. (2016). Transfer of location-specific control to untrained locations. Quarterly Journal of Experimental Psychology.

Wendt, M., Kluwe, R. H., \& Vietze, I. (2008). Location-specific versus hemisphere-specific adaptation of processing selectivity. Psychonomic Bulletin \& Review, 15(1), 135-140. doi:10.3758/ PBR.15.1.135

Wendt, M., \& Luna-Rodriguez, A. (2009). Conflict-frequency affects flanker interference: Role of stimulus-ensemble-specific practice and flanker-response contingencies. Experimental Psychology, 56(3), 206-217. doi:10.1027/1618-3169.56.3.206

Woodman, G. F., Vecera, S. P., \& Luck, S. J. (2003). Perceptual organization influences visual working memory. Psychonomic Bulletin \& Review, 10(1), 80-87.

Zurawska vel Grajewska, B., Sim, E.-J., Hoenig, K., Herrnberger, B., \& Kiefer, M. (2011). Mechanisms underlying flexible adaptation of cognitive control: Behavioral and neuroimaging evidence in a flanker task. Brain Research, 1421, 52-65. doi:10.1016/j.brainres.2011. 09.022 